\title{
Capturing the Real Impact of Clinical Academics in Practice
}

\author{
*Jane Coad ${ }^{\text {abcd }}$, Joseph C. Manning ${ }^{\mathrm{d}}$, Emma Mills ${ }^{\mathrm{e}}$, Cherith Semple ${ }^{\mathrm{f}}$, Bridget \\ Johnston $^{\text {gh }}$, and Ann McMahon ${ }^{\mathrm{i}}$ \\ ${ }^{a}$ University of Nottingham, United Kingdom; ${ }^{b}$ University Hospitals of Coventry and Warwickshire NHS \\ Trust, United Kingdom; ${ }^{\circ}$ Coventry University, United Kingdom; ${ }^{d}$ Nottingham University Hospitals NHS \\ Trust, United Kingdom; ${ }^{e}$ Bwrdd Iechyd Prifysgol Aneurin Bevan/Aneurin Bevan University Health Board, \\ United Kingdom; fUlster University / South Eastern Health \& Social Care Trust United Kingdom; \\ ${ }^{g}$ University of Glasgow, United Kingdom; ${ }^{h}$ NHS Greater Glasgow \& Clyde, United Kingdom; ${ }^{i}$ Royal \\ College of Nursing, UK, United Kingdom
}

\section{Abstract}

The Clinical Academic Careers Framework proposes an over-arching structure to develop the clinical academic workforce whose activities have patient benefit within a clear UK programme. Traditionally this has centred on professionals from medicine and dentistry, but in the last ten years has developed into a more inclusive career framework for non-medical health professions which includes Nurses, Midwives and Allied Health Professionals (NMAHPs) and Healthcare Scientists (HCSs) that provide NHS services. As such, it is reported that clinical academic NMAHPs and HCSs can contribute to the generation and translation of new knowledge to help improve outcomes and experiences for patients. In this article, we explore key issues relating to the impact of clinical academic NMAHPs and HCSs on clinical practice in a UK context, as well as some measurements of impact, including the value and limitations of currently used metrics (such as Key Performance Indicators, or KPIs). We report that measuring the learning in practice of this novel role will need to include smart metrics alongside a person-centred approach. We share four national case studies, all of which are drawn from clinical academic researchers from different UK settings to show the real variety and differences in roles. We argue that this is the key both to learning in practice about this role, and to witnessing the real differences clinical academics make.

\section{Keywords: clinical academic research careers; impact in practice; metrics of transformational change in practice}

\section{Introduction}

The Clinical Academic Careers Framework proposes an over-arching structure to develop the clinical academic workforce for patient benefit within a national consolidated programme. Traditionally, this has

*Corresponding Author: Professor Jane Coad, Professor of Children and Families Nursing, School of Health Sciences, Faculty of Medicine and Health Sciences, The University of Nottingham, Nottingham, NG7 2HA

\section{Email: jane.coad@nottingham.ac.uk}

Journal URL: https://publications.coventry.ac.uk/index.php/pblh

Coad, J., Manning, J. C., Mills, E., Semple, C., Johnston, B., \& McMahon, A. (2019). Capturing the Real Impact of Clinical Academics in Practice. International Journal of Practice-based Learning in Health and Social Care, 7(2), 47-56. DOI 10.18552/ijpblhsc.v7i2.647

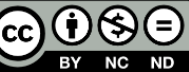

(C) 2019 Jane Coad, Joseph C. Manning, Emma Mills, Cherith Semple, Bridget Johnston, and Ann McMahon. This Open Access article is distributed under the terms of the Creative Commons Attribution $\begin{array}{lllll}\text { Attribution-Non-Commercial No Derivatives } & 4.0 & \text { International License }\end{array}$ (https://creativecommons.org/licenses/by-nc-nd/4.0/ ), which permits unrestricted non-commercial use, distribution, and reproduction in any medium, provided the original work is properly cited and is unaltered. 
centred on medical and dentist professionals. However, in the last ten years Clinical Academic Careers have rapidly developed in terms of a comprehensive research career framework for 'non-medical health professions', which includes Nurses, Midwives and Allied Health Professionals (NMAHPs) and Healthcare Scientists ( HCSs) working in the NHS or providing NHS services. It is reported that clinical academic researchers can contribute to the generation of new knowledge about care and treatment, whilst also seeking out the best evidence to help improve outcomes and experiences for patients (Carrick-Sen et al., 2016; Trueland, 2015; Westwood et al., 2013). This article highlights key issues relating to the impact of NMAHP and HCS clinical academics in the UK in terms of impact in real practice in order to discuss key learning issues.

\section{Background}

Globally, service users, commissioners of health and social care services, and research funders demand translational research (National Institute of Health Research, 2017). The emphasis is on delivering highest quality, clinical care where user involvement is paramount, alongside the growing need to base this on best evidence and research. Consequently, clinical academic NMAHPs and HCSs who hold posts both in health and social care organisations and associated academic higher education institutions [such as a university] are being regarded as the panacea to solve this challenge. There is now good evidence that they have made a demonstrable impact on quality of care and outcomes for patients, while improving the culture of working environment and so, in turn, the productivity of services.

In the UK, the Clinical Academic Roles and Career Pathways Implementation Network (CARIN) has been hosted by the UK Council of Deans since 2018 and was established to advise, support and share innovation and best practice of activity that promotes, engages and evaluates the development and implementation of joint clinical academic roles. Table 1 usefully sets out aspects of the current UK role.

\begin{tabular}{|ll|}
\hline - & Lead and contribute to new knowledge about care and treatment to improve patient outcomes. \\
\hline - & Advance practice and improve patient care through research \\
\hline - & Pursue evidence-based healthcare, improving quality, safety and effectiveness \\
\hline environment & $\begin{array}{l}\text { Contribute to a well-rounded clinical research community, supporting the development of a } \\
\text { healthcare workforce that actively seeks out the best evidence to help improve outcomes and } \\
\text { experiences for patients }\end{array}$ \\
\hline- & $\begin{array}{l}\text { Contribute to the health promotion and prevention agenda and support clinical decision- } \\
\text { making in partnership with patients }\end{array}$ \\
\hline- & Facilitate the adoption and spread of best practice, innovation and new technology \\
\hline - & $\begin{array}{l}\text { Contribute to the recruitment and retention of high-quality staff through increased } \\
\text { engagement, investment and support it }\end{array}$ \\
\hline
\end{tabular}

Table 1: Attributes of the UK clinical academic role (Association of UK University Hospitals, 2016)

However, the impact of clinical academic research and researchers is still to be realised. Furthermore, in some settings, these posts were and still are under-developed and opportunistically established as opposed to strategically planned. This is compounded by the fact that traditionally there was a lack of opportunities to access funding and fellowships along with a lack of confidence, skills and/or time to fully support the role (Hagan, 2018; Trueland, 2015). We would argue that healthcare providers can substantially benefit by incorporating clinical academic roles into healthcare organisations, but, from the outset, the organisational strategy needs to develop and agree the range of desired outcomes that should result from the development and employment of clinical academic researchers.

The question remains, how can NMAHPs and HCSs in clinical healthcare organisations work in partnership with Higher Education Institutions (HEIs) to maximise and benefit the goals of both 
organisations? In addressing this question, a clear strategy is firstly required with sign-up and agreements set in place. Any such strategy also needs to be based on strong reflective learning models in order for both the healthcare organisations and Higher Education Institutions to witness a positive and embracing cultural shift. In short, this can not solely rest on one or two individuals trying to make a change. Clearly, crucial factors for success are leadership, commitment and support but alongside it is essential that robust measurement needs to be set in place to capture the impact of NMAHP and HCS clinical academics and in turn the learning in practice of such roles.

\section{Measuring the impact}

In order to choose the best way to evaluate and judge the performance and progress of clinical academic NMAHPs and HCSs' impact on practice, there firstly needs to be a clear understanding of the core aims of this role. There are several complex levels to consider. For example, is it the person (individual level), strategy (organisational level), or patient intervention (research project) that actually makes a difference? We would argue that in reality there are numerous multi-levels, but we found little empirical evidence of these being inter-connected and no evidence which drew primarily from the patient's and stakeholder's perspectives of the real impact of this role. However, arguably these are still early days in this relatively new career framework.

Once outcomes have been agreed, measurement tools need to be decided upon but we would recommend they are robust, relevant and focused on or aligned with the strategy. The more the measurements are in tune with what clinical professionals do, the more they will be viewed as useful and meaningful, and thus provide a greater drive for the strategy of having clinical academic researchers leading clinical and service innovation and improvements (Better Care Fund, 2015). The timeframe over which such outcomes can reasonably be expected to be achieved is also important. We would suggest a realistic timeframe is three to five years from outset through to witnessing real tangible results of impact and change in practice. This is key to understanding that investment in professional clinical staff is not a quick gain but more of a long-term mission.

Metrics for recording learning change in practice can also include the use of Key Performance Indicators (KPIs) to measure what an individual, department or organisation uses to define success and to track progress to meet strategic goals (Association of UK University Hospitals, 2016). This useful approach for organisations seeks to support transformational healthcare though the introduction of clinical academic roles in NMAHPs and HCSs. Accepting the wisdom that macro, meso and micro levels of influence are at play at organisational, unit and individual levels, it is worth reiterating that the ability of an individual to attain given KPIs within the context of a clinical academic role is contingent on the conditions within the unit and the organisation where they work. We would argue that without a clear understanding of collective responsibility, the ambitions set out above are unlikely to happen. For example, when considering the aspirations set out in the table (Table 2), typical KPIs may include:

- Secure grant income to cover salary costs

- Publish two papers in peer-reviewed journals annually

- $\quad$ Provide two examples of research that has been implemented and that has improved clinical practice

- $\quad$ Provide two examples of where quality, safety and effectiveness have improved based on the best available evidence

- Supervise two staff undertaking a research Masters dissertation in clinical practice

- Support two staff in leading a service innovation / improvement in clinical practice

- Support two patient-empowerment projects in clinical practice

- Support one project exploring the potential of digital innovation in clinical practice

- Demonstrate and monetise a contribution to staff retention

Table 2: Aspirations of Key Performance Indicators 
There are two things to note here. The first is that many of these suggested KPIs may be merged and the list significantly collapsed. The second is that the KPIs serve to illustrate the necessity for the entire team to work together to make any of these ambitions achievable. For example, a comprehensive understanding of the personal time and investment required to secure grant income and publish papers is essential for any one individual to achieve these KPIs. Above all, however, these measures are only what they claim to be - indicators. Transformation, or metamorphosis, is far more subtle and complex than KPIs might suggest.

\section{Real learning from practice}

Consequently, we are suggesting here that measuring real learning in practice is not always about metrics. It is about how we observe the types of knowledge and skills that clinical academics possess, and can bring to bear, which then impact on clinical practice. This may be more subtle learning in practice and may not relate to mechanism and outcome-type metrics. It is about a much more person-centred change and what collectively those individuals can do which in turn will drive improvements in the culture of the organisation and in quality of care. In order to highlight real exemplars of clinical academics who are making a difference in their roles, we have collated case studies using a four-country UK perspective.

\section{Case Study 1 - England:}

\section{Dr Joseph Manning, NIHR/HEE ICA Clinical Lecturer; Clinical Associate Professor in Children, Young People and Families Nursing; Charge Nurse, Paediatric Critical Care Outreach, Nottingham University Hospitals NHS Trust and The University of Nottingham}

In my current role, I work across a large tertiary NHS Trust and a university with a clinical, research and leadership remit. The NHS Trust, Nottingham University Hospitals NHS Trust, is one of the largest acute trusts in the UK, providing a huge variety of specialist services (including a children's hospital, tertiary neonatal services, and a regional trauma centre) to over 2.5 million children, young people and adults that reside in Nottingham and the surrounding communities. It is one of the largest employers in the region, with around 16,700 staff, of whom over half are NMAHPs and HCSs.

Specifically, my role includes clinical care delivery as a charge nurse for critical care outreach, leading the development of capacity and capability of NMAHPs in the children's hospital, and developing a programme of research aims to build understanding of: (1) the holistic needs of children and families in the short- and long-term after critical illness; (2) the emotional health and wellbeing of children and young people admitted to acute paediatric inpatient care; and (3) the safe and effective transitions of infants, children and young people between services and pathways of care.

At a patient level the impact of my clinical-academic role is two-fold. My clinical practice involves timely and expert recognition and treatment of the deteriorating child or young person. This has a direct impact on patients' quality of life, experience and outcomes by supporting staff with more complex procedures, facilitating escalation of treatment, supporting capacity and service delivery through assisting with communications across the Multi-Disciplinary Team, and enhancing the learning environment by educating ward staff at the bedside in the care and management of the sick child. Moreover, my programme of research is driven by the clinical insights from my practice and consultation with the Multi-Disciplinary Team with whom I work closely. This research has demonstrable impact on the quality and outcomes of the children and families who access our services. An example of this relates to the growing numbers of children and young people accessing acute paediatric care in mental health crisis with local stakeholder feedback indicating that both patient experience and outcomes required improvement. This has contributed to driving forward clinical care through understanding stakeholders' perspectives (Manning et al., 2015), developing and testing of effective educational resources for staff (Manning et al., 2017), and the development of tools to support staff to make decisions regarding the safety of these children and young people (Carter et al., 2019; Manning et al., 2018).

At an organisational level, my developing programme of research, which is aligned to our clinical care, contributes to the overarching ambition of the healthcare provider as a research-intensive organisation as 
well as its commitment to advancing care through research and innovation. I support the building of capacity for NMAHPs to develop research with children and families. This has involved extensive coaching and role modelling at individual, service and department levels as well as working with managers and leaders to develop understanding around the value and impact of clinical-academic roles. As a senior nurse, I am able to influence and be a catalyst for harnessing a workplace culture that values research and evidence-based practice. The clinical-academic skillset and insight that I bring affords a unique vantage point to appraise specific problems that healthcare organisations are contending with and to identify innovative and sustainable solutions. An example of this has been the chief nurse fellow initiative, which has provided an opportunity for frontline staff to innovate and develop their skills whilst remaining in practice (Bramley et al., 2018).

\section{Case Study 2 - Scotland:}

\section{Professor Bridget Johnston, Professor/Florence Nightingale Foundation Chair in Clinical Nursing, University of Glasgow and NHS Greater Glasgow \& Clyde, UK}

I have a clinical, education and research background in palliative and end of life care and the majority of my clinical role revolves around issues to do with palliative, end of life care and bereavement. My role is unique for a nursing professor - not just in the UK but internationally - in that I am $50 \%$ university and $50 \%$ NHS based. NHS Greater Glasgow and Clyde is the largest health board in Scotland and serves a population of 1.14 million with 11,675 Registered Nurses, 2,730 registered Allied Health Professionals and 630 Midwives. There are 35 hospitals of different types including one of the largest acute hospitals in the UK. There are six adult hospices and one children's hospice in the Greater Glasgow and Clyde area. My specialism is palliative, end of life care and bereavement which is an important area for health care in general and not just nursing. Palliative and end of life care needs are experienced by large numbers of people living with, and dying from, life-limiting conditions in home, hospice, care home and hospices. I have specialised in palliative and end of life care for over 30 years, and currently my clinical practice also focuses on bereavement care and support.

A key aspect needed to develop my role has been role models and mentors in practice. Choosing to follow a clinical academic path is a rewarding but challenging task, particularly in terms of balancing clinical and academic responsibilities and responding to two different managers. Education and training are also important for this role. These include skills development; in my role, excellent communication skills are necessary as well as leadership skills. In order to lead funded research projects and to develop a team, skills in both the research area and research methods are imperative. Impact being embedded in practice is one of the most important aspects of my job. Changing, improving and supporting practice is much harder when only an academic institution employs you. As a nurse, you are always stronger alongside other nurses and clinical team members, so everything I have achieved has been in conjunction with clinical colleagues.

Some recent achievements I have made in practice relate to bereavement care. Across all of the acute hospitals in Greater Glasgow and Clyde, we have replaced property bags (for bereaved relatives after their loved one has died) with hessian bags and organza bags for jewellery. We have had positive feedback from family members regarding the difference this has made. This exemplifies how small changes can make a big difference.

Another target we have is to improve communication skills and support for staff. In particular, we are aiming to provide skills training and support for frontline non-clinical staff and we plan in the next few months to provide a day of training in managing distress in patients and relatives, and emotional support for ward reception staff and staff working in our mortuaries. I have made the assumption that, in order to improve end of life care in acute hospitals, we need to listen to people dying (if they are able to communicate), as well as their bereaved family and friends. We have recently completed a 12-month pilot project across Glasgow to gain the views of relatives; we are collating feedback and will make changes and improvements in care where appropriate. We are also planning to test different methods to find the best way of gaining feedback from people and relatives regarding end of life care in hospital. 
In terms of making a difference to patient outcomes, I have been able, working closely with clinical teams and by role modelling, to improve bereavement care in our acute hospitals. We have also improved end of life care by responding to - and learning from - patient and family feedback We have also improved end of life care by carrying out a specific evidenced-based end of life intervention: 'dignity therapy'. We are running this as a clinical project and not research, but have patient and family feedback on outcomes in terms of how much patients and families have benefited from the intervention. In turn, this all serves to improve knowledge on evidenced-based practice as well as specifically developing skills, confidence and understanding of bereavement care and end of life care. Moreover, my programme of research, developing and testing interventions in palliative and end of life care and bereavement, aligns with our organisation goals on clinical care. In addition, as a member of the senior (chief nurse) team, I am able to influence, provide strategic direction and enable a culture that values research in our NMAHP workforce.

\section{Case Study 3-Wales:}

\section{Emma Mills - Consultant Midwife/ Lead Clinical Research Midwife, Bwrdd lechyd Prifysgol Aneurin Bevan/Aneurin Bevan University Health Board, UK}

I have been a qualified Registered Nurse and Midwife for almost twenty-eight years. After studying for an MSc in reproduction and health, I developed a passion for research, but stayed in a clinical role as I believed at the time it was where I could make the most difference to women and their families. However, when a part-time role implementing a midwifery research study became available, I decided that I would be able to combine both research and clinical work. At this point the maternity services of Bwrdd Iechyd Prifysgol Aneurin Bevan had supported occasional research studies but there was no dedicated research midwife and therefore limited opportunities for the service to be expanded. At the same time all Health Boards in Wales became University Health Boards and the need for commitment to clinical research was highlighted. Currently, Aneurin Bevan University Health Board covers a large geographical area in South Wales, including the areas of Blaenau Gwent, Caerphilly, Monmouthshire, Newport, Torfaen and South Powys. The Health Board employs over 13,000 staff, two-thirds of whom are involved in direct patient care. There are 4,908 NMAHPs and 250 Clinical Health Scientists.

When I started my clinical academic career, I felt I really needed to observe and learn from good leadership and from others who were on a clinical academic research pathway. Whilst I could identify individuals nationally, at a local level I found that I needed a supportive research and development clinical department with strong links with the local university, Health and Care Research Wales, and Welsh Government. In turn, this ensured a successful transition into a research-active maternity department and a positive research culture.

In terms of impact, I very quickly realised not only how much I loved coordinating clinical trials and how engaged women were at contributing to improving national midwifery care through research, but also how it could have an impact both directly and indirectly on clinical care. I was able to spend a lot of time talking to families about their experiences, with sometimes heart-breaking situations such as stillbirth and premature labour, but also their passions and enthusiasm about contributing to something that could make this better for other families.

In terms of a difference, I feel that in this role I have been able to enthuse and motivate staff, offering opportunities for other midwives to become involved in research and service improvement projects. The research culture within our health board maternity services has become so positive; it is almost unrecognisable, through staff members taking pride and ownership in making a difference to families and being the best that they can. After publishing my own piece of research (Mills, 2018), following a Building Research Capacity for Nursing and Allied Health Professionals (RCBC) scholarship, I have been able to support two other midwives in applying for the same assistance, also supporting them to write and develop studies that aim to improve care and explore patient experience. Since gaining research experience, I have been able to support other midwives in submitting abstracts and presenting at national conferences, putting forward ideas and initiatives, and we have reached record levels of staff who are Good Clinical Practice (GCP) research trained. Overall, staff members say they feel encouraged to take ownership in demonstrating that our department is progressive, up to date and contributing to the body of evidence at local, national and international levels. Since beginning contributing to this article, I have 
become a Consultant Midwife, a role with a strong research focus, and one where I believe I can continue to support colleagues and women to recognise the importance of research in clinical practice.

\section{Case Study 4 - Northern Ireland:}

\section{Dr Cherith Semple, Reader in Clinical Cancer Nursing, Ulster University / South Eastern Health \& Social Care Trust (SEHSCT)}

My current role, as a Reader in Clinical Cancer Nursing provides a unique clinical and research interface, as I work collaboratively across a health and social care trust (SEHSCT, 0.6WTE) and an academic institution (Ulster University, 0.4 WTE) in Northern Ireland. The SEHSCT is one of five integrated health and social care trusts in Northern Ireland, incorporating acute hospital services, community health and social care services; it serves a population of 361,000 people, with approximately 2,800 Registered Nurses and Midwives, and 630 Allied Health Professionals.

My area of clinical expertise is head and neck cancer. This clinical academic role enables me to embed direct clinical care, having a caseload of patients newly diagnosed with head and neck cancer, while embracing research and innovation to drive improvements in care through two programmes of research. These programmes of research aim: 1) to promote post-treatment quality of life for patients with head and neck cancer, and 2) to promote positive coping for families impacted by parental cancer - 'Familycentred cancer care'.

On obtaining a Doctoral Fellowship, which led to the completion of my PhD in 2006, I was keen to pursue a career that allowed me to deliver direct patient care but with capacity to focus simultaneously on generating evidence, which would lead to improvements in the quality and efficiency of care delivered to patients with cancer. My current clinical academic research role came to fruition in 2018, but required clear, strategic organisational vision between Ulster University and South Eastern Health \& Social Care Trust (SEHSCT), with good partnership working, and clear and transparent processes for accountability and communication. Personally, it was important to have expert clinical leadership, build upon collaborative research networks, have mentorship and be tenacious with realistic goals clearly outlined within a workplan.

Such innovative partnership roles will not only enable a generation of clinically relevant research questions but facilitate linkage to research infrastructure, such as statistical support that promotes robust research studies being conducted. One further benefit of this environment is the timely integration and translation of findings into clinical practice at the point of care, therefore instrumental in ensuring the implementation of best practice and innovation. These roles also maximise opportunities for supporting clinical colleagues to develop their research skills and establish a culture of enquiry and researchmindedness. Furthermore, I have found that working within an establishing Cancer Care Research Group at Ulster University prevents isolation, maximises shared learning, and promotes thematic programmes of research.

In addition, these roles create capacity to supervise Masters' Degree level and Doctoral students, to impact teaching programmes for nurses such a short course on head and neck cancer management, and to contribute to the core-trainee programme for dentists. Another important aspect is mentoring nurses, which is especially pertinent as the cancer nursing workforce expands. These roles also mean there is a real opportunity to work across professional and organisational boundaries. For example, I am currently leading on two research projects with dentistry colleagues to improve service delivery for patients who require interventions such as multiple dental extractions and obturator prosthesis as a consequence of head and neck cancer treatment, while collaborating and networking with Ear Nose and Throat (ENT) colleagues in Nordic countries. Within the family-centred cancer care programme of work, an evidencebased e-learning module has been developed to equip healthcare professionals with the necessary tools and strategies to empower parents to communicate their cancer diagnosis with children. This is freely available at http://training.cancerfocus.org. Finally, but of great importance, these roles create immense job satisfaction, allowing for creativity which helps build resilience. 


\section{Key learning points}

The points raised in the article and the four cases highlight a number of issues. First and foremost, the NHS healthcare environment remains very challenging and, although several external challenges can also provide an opportunity to transform and improve the design, delivery, safety, patient benefit and satisfaction of health services, the constrained financial environment and commissioner affordability are key risks. One learning point from all the cases is the need to foster strong partnerships and positive strategic alliances. Investment at one level should arguably reward the organisation at another.

All of the cases highlight the need for strategic vision which transcends the clinical area and higher education institution (often this is a local arrangement). Drawing on one example where the lead author (Coad) works, University Hospital Coventry \& Warwickshire NHS Trust's (UHCW) vision is to become a national and internal leader in healthcare. Both Research and Development are essential components of world-leading excellence in clinical care in order that our patients receive the very best care possible. Working in collaboration, since 2018, both Research \& Development (Head of Research, Ceri Jones; Chief Nurse, Nina Morgan; Director of Education and Research, Vicky Williams and Professor Chris Imray) at UHCW and the lead author (Coad) are now setting out an ambitious strategy for 2018-2021, to be known as the Interdisciplinary Clinical Academic Health Research Excellence (i-CAhRE) Programme, for all Nurses, Midwives, Allied Health Professionals and Clinical Health Scientists in terms of their clinical academic research careers (See: https://www.uhcw.nhs.uk/leading-research/about-us/ourresearchers/). The overall aims of the iCAhRE programme are:

(1) To develop capacity and capability through developing and embedding clinical academic research careers and roles across inter-disciplinary groups in the Trust;

(2) To develop opportunities for research training and education for clinical academic researchers;

(3) To develop a supportive and empowering infrastructure alongside embedding an innovative interdisciplinary clinical academic research culture across the Trust.

This innovative strategy will enable UHCW staff to develop clinical academic research careers and will contribute to the generation of new knowledge which will improve outcomes and experiences for patients. The team has spent a year developing the strategy which was important not only to set out the strategy but to agree how this would work at the organisational level. We have ensured senior management, including the Chief Nurse and Chief Executive, have agreed the vision and have set out a plan to support managers in understanding the benefit of having such roles in the organisation and the links to local HEI providers (such as Coventry University; University of Warwick, University of Birmingham and University of Nottingham). The vision includes the appointment of research leaders and a supportive team in order to ensure excellent support, good governance and long-term sustainability.

In initiating the vision, we have developed a dedicated Patient and Public Involvement and Engagement (PPIE) strategy where all Clinical Academic Researchers will discuss and agree their research or careers at the outset and throughout any study and/or Fellowship. To date, activities have included support for application and expansion of relevant research and education opportunities including our Doctorate portfolio. Success in one year (2018-2019) has totalled over £1.6 million to UHCW which is an outstanding metric along with other excellent success such as NIHR funding, two NHS $70 @ 70$ leadership awards and a rising number of outputs and accolades.

We have drawn together a number of individual cases and one organisational vision in transforming clinical academic research to enable transformational practice. Whilst this is an exciting time it highlights how important strategic oversight and engagement is in terms of developing the clinical academic researcher role. It also highlights that impact can only be achieved in a supportive organisation and can be very challenging for those working alone and/or who are not supported by their employing organisation. An individual can lead clinical academic research for their projects and organisation but it is the collective partnerships, solid investment and joined-up strategy that are required to really make demonstrable changes and measurable impacts in practice. 


\section{Discussion and conclusion}

We have set out an article to demonstrate the importance of strategic and operational planning that is required in order to develop the clinical academic researcher role for NMAHPs and HCSs. We have suggested that Key Performance Indicators are simply not enough and that more subtle insight is required to surface the complexity of impact in practice illustrated through our personal case studies and experiences drawn from across the UK.

Using learning from real-world examples, we have outlined that clinical academic researchers must be astute and must select meaningful metrics to demonstrate impact whilst also capturing the relational, the humanistic, and the person-centred change that is enacted through their roles and activities. Measuring the learning in practice of this novel role will need to include smart metrics alongside a person-centred approach. We have included both functions here in this article, and have used case studies from different UK settings to show the real variety and differences in clinical academic research roles. We can conclude that the true key to learning about clinical academic research roles is witnessing the real differences clinical academics make to practice when given the opportunities and positive organisational culture to do so.

\section{ORCID}

Jane Coad

Joseph C. Manning

Cherith Semple

Bridget Johnston https://orcid.org/0000-0002-1043-5935

https://orcid.org/0000-0002-6077-4169

https://orcid.org/0000-0002-4560-7637

https://orcid.org/0000-0003-4051-3436

\section{References}

Better Care Fund. (2015). How to understand and measure impact. NHS. https://www.england.nhs.uk/wp-content/uploads/2015/06/bcf-user-guide-04.pdf.pdf

Bramley, L., Manning, J., \& Cooper, J. (2018). Engaging and developing frontline clinical nurses to drive care excellence: Evaluating the Chief Nurse Excellence in Care Junior Fellowship initiative. Journal of Research in Nursing, 23(8), 678-689. https://doi.org/10.1177/1744987118808843

Carrick-Sen, D., Richardson, A., Moore, A., \& Dolan, S. (2016). Transforming healthcare through clinical academic roles in nursing, midwifery and allied health professions: A practical resource for healthcare provider organisations. https://councilofdeans.org.uk/wpcontent/uploads/2019/02/AUKUH-Transforming-Healthcare.pdf

Carter, T., Walker, G. M., Aubeeluck, A., \& Manning, J. C. (2019). Assessment tools of immediate risk of self-harm and suicide in children and young people: A scoping review. Journal of Child Health Care, 23(2), 178-199. https://doi.org/10.1177/1367493518787925

Hagan, J. (2018). Nurse satisfaction with opportunities to engage in research. Western Journal of Nursing Research, 40(2), 209-221. https://doi.org/10.1177/0193945916682472

Manning, J. C., Bean, D., Coad, J., Blake, I., Bingham, V., Cooper, J., Carter, T., Turner, A., Hendron, E., Michail, M., Rychwalska Brown, L., Wood, D., \& Latter, K. (2015). Evaluating quality and impact of acute paediatric inpatient care: Defining the domains for a Person Centred Outcome Measure (PCOM) in children and young people admitted with self-harm or eating disorders. Nottingham University Hospitals NHS Trust.

Manning, J. C., Carter, T., Latif, A., Horsley, A., Cooper, J., Armstrong, M., Crew, J., Wood, D., Callaghan, P., \& Wharrad, H. (2017). 'Our Care through Our Eyes'. Impact of a co-produced digital educational programme on nurses' knowledge, confidence and attitudes in providing care 
for children and young people who have self-harmed: A mixed-methods study in the UK. BMJ Open, 7(4), e014750. https://bmjopen.bmj.com/content/7/4/e014750

Manning, J. C., Walker, G. M., Carter, T., Aubeeluck, A., Witchell, M., \& Coad, J. (2018). Children and Young People-Mental Health Safety Assessment Tool (CYP-MH SAT) study: Protocol for the development and psychometric evaluation of an assessment tool to identify immediate risk of self-harm and suicide in children and young people (10-19 years) in acute paediatric hospital settings. BMJ Open, 8(4), e020964. https://doi.org/10.1136/bmjopen-2017-020964

Mills, E. (2018). UK midwives' knowledge and understanding of female genital mutilation. MIDIRS Midwifery Digest, 28(4), 491-496.

National Institute of Health Research. (2017). NIHR Annual report: Improving the health and wealth of the nation through research. https://www.nihr.ac.uk/documents/about-us/our-contribution-toresearch/research-performance/NIHR-Annual-Report-2017-18.pdf

Trueland, J. (2015, November 18). Birth of a new breed: Interview with Dave Jones. The Health Service Journal, 2-3. https://www.hsj.co.uk/Uploads/y/r/i/Research--Impact-supplement-18th-Nov2015.pdf

Westwood, G., Fader, M., Roberts, L., Green, S. M., Prieto, J., \& Bayliss-Pratt, L. (2013). How clinical academics are transforming patient care. Health Service Journal, 123(6368), 28-29. 